\title{
ABSTRACT KEY POLYNOMIALS AND COMPARISON THEOREMS WITH THE KEY POLYNOMIALS OF MAC LANE - VAQUIÉ
}

\author{
J. Decaup ${ }^{1}$, W. Mahboub ${ }^{2}$, M. Spivakovsky ${ }^{1}$ \\ 1 Institut de Mathématiques de Toulouse/CNRS UMR 5219, Université Paul Sabatier, 118, \\ route de Narbonne, 31062 Toulouse cedex 9, France. \\ ${ }^{2}$ Department of Mathematics, American University of Beirut.
}

\section{INTRODUCTION}

Let $K$ be a valued field and $K \hookrightarrow K(x)$ a simple purely transcendental extension of $K$. In the nineteen thirties, $\mathrm{S}$. Mac Lane considered the special case when the valuation $\nu$ of $K$ is disrete of rank one and defined the notion of key polynomials, associated to various extensions of $\nu$ to $K(x)$ ([3] and [4]). Key polynomials are elements of $K[x]$ which describe the structure of various extensions of $\mu$ to $K(x)$ and the relationship between them. Roughly speaking, they measure how far a given extension of $\mu$ to $K(x)$ is from the monomial valuation (the one that assigns to each polynomial $f \in K[x]$ the minimal value of the monomials appearing in $f$ ). Mac Lane's definition of key polynomials was axiomatic: an element $f \in$ $K[x]$ is a key polynomial for an extension $\mu$ of $\nu$ to $K$ if it is monic, $\mu$-minimal and $\mu$ irreducible (see Section 2 below for precise definitions).

Michel Vaquié ([8], [9], [10] and [11]) extended this definition to the case of arbitrary valued fields $K$ (that is, without the assumption that $\nu$ is discrete). One important difference with the case of discrete valuations treated by Mac Lane is the presence of limit key polynomials (which will not be discussed in the present paper).

F. H. Herrera, M. A. Olalla, W. Mahboub and M. Spivakovsky defined a different, though closely related notion of key polynomials ([1] and [2]). In their approach the emphasis was on describing key polynomials by explicit formulae and on constructing the successive key polynomials recursively in terms of the preceding ones.

In his Ph.D. thesis (Toulouse 2013), W. Mahboub proved comparison theorems between Mac Lane - Vaquié key polynomials.

Apart from a better understanding of the structure of simple extensions of valued fields in its own right, one of the intended applications of the theory of key polynomials is the work towards the proof of the Local Uniformization Theorem over fields of arbitrary characteristic. Jean-Christophe San Saturnino (see Theorem 6.5 of [7]) proved that in order to achieve Local Uniformization of a variety embedded in Spec $k\left[u_{1}, \ldots, u_{n}\right]$ along a given valuation $\mu$ of $k\left(u_{1}, \ldots, u_{n}\right)$ it is sufficient to monomialize the first limit key polynomial of the simple extension $k\left(u_{1}, \ldots, u_{n-1}\right) \hookrightarrow k\left(u_{1}, \ldots, u_{n}\right)$ (assuming local uniformization is already known in ambient dimension at most $n-1)$. Although limit key polynomials are beyond the scope of this paper, we hope that the comparison theorems proved here will clarify the relationship between different definitions of key polynomials and therefore be useful for applications.

Let $\mu$ be an extension of $\nu$ to $K$. In this paper we give a new definition of key polynomials (which we call abstract key polynomials) associated to $\mu$ and study the relationship 
between them and key polynomials of Mac Lane - Vaquié. Associated to each abstract key polynomial $Q$, we define the truncation $\mu_{Q}$ of $\mu$ with respect to $Q$. Roughly speaking, $\mu_{Q}$ is an approximation to $\mu$ defined by $Q$. This approximation gets better as $\operatorname{deg}_{x} Q$ and $\mu(Q)$ increase. We also define the notion of an abstract key polynomial $Q^{\prime}$ being an immediate successor of another abstract key polynomial $Q$ (in this situation we write $Q<Q^{\prime}$ ). The main comparison results proved in this paper are as follows:

Theorem 23. An abstract key polynomial for $\mu$ is a Mac Lane - Vaquié key polynomial for the truncated valuation $\mu_{Q}$.

Theorem 26: If $Q<Q^{\prime}$ are two abstract key polynomials for $\mu$ then $Q^{\prime}$ is a Mac Lane Vaquié key polynomial for $\mu_{Q}$.

Theorem 27] which, for a monic polynomial $Q \in K[x]$ and a valuation $\mu^{\prime}$ of $K(x)$, gives a sufficient condition for $Q$ to be an abstract key polynomial for $\mu^{\prime}$. Combined with Proposition 1.3 of [9], this describes a class of pairs of valuations $\left(\mu, \mu^{\prime}\right)$ such that $Q$ is a Mac Lane - Vaquié key polynomial for $\mu$ and an abstract key polynomial for $\mu^{\prime}$. This can be regarded as a partial converse to Theorem 26 .

This paper is structured as follows. In \$2 we define the Mac Lane - Vaquié and the abstract key polynomials and study their properties. In 33 we prove our main comparison results stated above.

\section{Preliminaries AND NOTAtion}

Throughout this paper, $\mathbb{N}$ will denote the non-negative integers, $\mathbb{N}^{*}$ the strictly positive integers. For a field $L$, the notation $L^{*}$ will stand for the multiplicative group $L \backslash\{0\}$.

- Let $R$ be a domain, $K$ the field of fractions of $R, \mu$ a valuation of $K$ with value group $\Gamma$ and $\alpha \in \Gamma$. We define:

(1) $\mathcal{P}_{\alpha}(R):=\{x \in R$ such that $\mu(x) \geq \alpha\}$

(2) $\mathcal{P}_{\alpha^{+}}(R):=\{x \in R$ such that $\mu(x)>\alpha\}$

(3) $\operatorname{gr}_{\mu}(\mathrm{R}):=\bigoplus_{\alpha \in \Gamma} \frac{\mathcal{P}_{\alpha}(\mathrm{R})}{\mathcal{P}_{\alpha}+(\mathrm{R})}$

(4) $G_{\mu}:=\operatorname{gr}_{\mu}(K)$

(5) For each $f \in R$ such that $\mu(f)=\alpha$, we denote by $\operatorname{in}_{\mu}(f)$ the image of $f$ in $\frac{\mathcal{P}_{\alpha}(R)}{\mathcal{P}_{\alpha^{+}}(R)}$; we call this image the initial form of $f$ with respect to $R$ and $\mu$.

- Let $K \hookrightarrow K(x)$ be a purely transcendental extension of $K$. Let $Q$ be a monic polynomial in $K[x]$. Every polynomial $g \in K[x]$ can be written in a unique way as

$$
g=\sum_{j=0}^{s} g_{j} Q^{j}
$$

with all the $g_{j} \in K[x]$ of degree strictly less than $\operatorname{deg}(Q)$. We call (1.1) the $Q$ expansion of $g$.

Definition 1. Let $g=\sum_{j=0}^{s} g_{j} Q^{j}$ be the $Q$-expansion of an element $g \in K[x]$. We put $\mu_{Q}(g):=\min _{\substack{0 \leq j \leq s \\ g_{j} \neq 0}} \mu\left(g_{j} Q^{j}\right)$ and we call $\mu_{Q}$ the truncation of $\mu$ with respect to $Q$.

- Let $\mu$ be a valuation of the field $K(x)$, where $x$ is an algebraically independent element over a field $K$. Consider the restriction of $\mu$ to $K[x]$. Consider a monic polynomial $Q \in K[x]$. Assume that $\mu_{Q}$ is a valuation (below we will define the notion of abstract key polynomial and will show that $\mu_{Q}$ is always a valuation in that case). Fix another 
polynomial $f$ and let $f=\sum_{j=0}^{s} f_{j} Q^{j} \in K[x]$ be the $Q$-expansion of $f$. Let $\alpha=\mu_{Q}(f)$.

We denote by $\operatorname{In}_{Q} f$ the element $\sum_{\mu\left(f_{j} Q^{j}\right)=\alpha} f_{j} Q^{j} \in K[x]$. Note that, by definition, $\operatorname{In}_{Q} f \in$ $K[x]$, while $\operatorname{in}_{\mu_{Q}} f \in \operatorname{gr}_{\mu_{Q}} K[x]$. We have $\operatorname{in}_{\mu_{Q}}\left(\operatorname{In}_{Q} f\right)=\operatorname{in}_{\mu_{Q}} f$.

\section{Key Polynomials}

2.1. Key polynomials of Mac Lane-Vaquié. We first recall the notion of key polynomial, introduced by Vaquié in [9], generalizing an earlier construction of Mac Lane [4].

Definition 2. Let $(f, g) \in K[x]^{2}$. We say that $f$ and $g$ are $\mu$-equivalent and we write $f \sim_{\mu} g$ if $f$ and $g$ have the same initial form with respect to $K$ and $\mu$.

Remark 3. The polynomials $f$ and $g$ are $\mu$-equivalent if and only if

$$
\mu(f-g)>\mu(f)=\mu(g) .
$$

Indeed, if

$$
\operatorname{in}_{\mu} f=\operatorname{in}_{\mu} g
$$

then, in particular, $\mu(f)=\mu(g)$. Furthermore, (2.1) says that $f$ and $g$ agree modulo $\mathcal{P}_{\mu(f)^{+}}$. Thus

$$
\mu(f-g)>\mu(f)=\mu(g) .
$$

Conversely, if $\mu(f-g)>\mu(g)$, then $\operatorname{in}_{\mu}(f-g+g)=\operatorname{in}_{\mu}(g)$.

Definition 4. Let $(f, g) \in K[x]^{2}$. We say that $g$ is $\mu$-divisible by $f$ or that $f \mu$-divides $g$ (denoted by $\left.f\right|_{\mu} g$ ) if the initial form of $g$ with respect to $\mu$ is divisible by the initial form of $f$ with respect to $\mu$ in $\operatorname{gr}_{\mu} K[x]$.

Remark 5. We have $\left.f\right|_{\mu} g$ if and only if there exists $c \in K[x]$ such that $g \sim_{\mu} f c$.

Definition 6. Let $Q \in K[x]$ be a monic polynomial. We say that $Q$ is a Mac Lane-Vaquié key polynomial for the valuation $\mu$ if the following conditions hold:

(1) $Q$ is $\mu$-irreducible, that is, for any $g, h \in K[x]$, if $\left.Q\right|_{\mu} g h$, then $\left.Q\right|_{\mu} g$ or $\left.Q\right|_{\mu} h$.

(2) $Q$ is $\mu$-minimal, that is, for every $f \in K[x]$, if $\left.Q\right|_{\mu} f$ then $\operatorname{deg}(f) \geq \operatorname{deg}(Q)$.

Proposition 7. Let $P$ be an element of $K[x]$. Assume that $P$ is $\mu$-irreducible. Then $\operatorname{in}_{\mu} P$ is irreducible in $\operatorname{gr}_{\mu} K[x]$.

Proof. Assume that $\operatorname{in}_{\mu} P$ is reducible in $\operatorname{gr}_{\mu} K[x]$, aiming for contradiction. Write $\operatorname{in}_{\mu} P=$ $\operatorname{in}_{\mu} g$ in $_{\mu} h$ with $\mu(g), \mu(h)>0$.

We have $\left.P\right|_{\mu} g h$, but $P \nmid_{\mu} g$ and $P \nmid_{\mu} h$. This contradicts the $\mu$-irreducibility of $P$. The Proposition is proved.

Remark 8. Assume that every homogeneous element of $\operatorname{gr}_{\mu} K[x]$ admits a unique decomposition into irreducible factors. Then $Q$ is $\mu$-irreducible if and only if its initial form with respect to $\mu$ is irreducible.

We now introduce an alternative, though closely related notion of key polynomials. 
2.2. Abstract key polynomials. We keep the same notation as in 2.1, and we add the following:

(1) For each strictly positive integer $b$, we write $\partial_{b}:=\frac{\partial^{b}}{b ! \partial x^{b}}$, the so-called $b$-th formal derivative with respect to $x$.

(2) For each polynomial $P \in K[x]$, let $\epsilon_{\mu}(P):=\max _{b \in \mathbb{N}^{*}}\left\{\frac{\mu(P)-\mu\left(\partial_{b} P\right)}{b}\right\}$

(3) For each polynomial $P \in K[x]$, let $b(P):=\min I(P)$ where

$$
I(P):=\left\{b \in \mathbb{N}^{*} \text { such that } \frac{\mu(P)-\mu\left(\partial_{b} P\right)}{b}=\epsilon_{\mu}(P)\right\} .
$$

Definition 9. Let $Q$ be a monic polynomial in $K[x]$. We say that $Q$ is an abstract key polynomial for $\mu$ if for each polynomial $f$ satisfying

$$
\epsilon_{\mu}(f) \geq \epsilon_{\mu}(Q)
$$

we have $\operatorname{deg}(f) \geq \operatorname{deg}(Q)$.

Proposition 10. Let $t \geq 2$ be an integer, $Q$ an abstract key polynomial and $P_{1}, \ldots, P_{t} \in K[x]$ of degrees strictly less than $\operatorname{deg}(Q)$. Let $\prod_{i=1}^{t} P_{i}=q Q+r$ be the Euclidean division of $\prod_{i=1}^{t} P_{i}$ by Q. Then

$$
\mu(r)=\mu\left(\prod_{i=1}^{t} P_{i}\right)<\mu(q Q)
$$

Proof. We proceed by induction on $t$.

First, consider the case $t=2$. We want to show that

$$
\mu\left(P_{1} P_{2}\right)=\mu(r)<\mu(q Q) .
$$

Assume the contrary, that is, $\mu\left(P_{1} P_{2}\right) \geq \mu(q Q)$ and $\mu(r) \geq \mu(q Q)$. For each $j \in \mathbb{N}^{*}$, we have $\mu\left(\partial_{j} P_{1}\right)>\mu\left(P_{1}\right)-j \epsilon_{\mu}(Q)$, and similarly for $P_{2}, q, r$, because all these polynomials have degree strictly less than $\operatorname{deg}(Q)$ and $Q$ is an abstract key polynomial. Since $\mu\left(\partial_{j} q\right)>\mu(q)-j \epsilon_{\mu}(Q)$ for all strictly positive integers $j$, we deduce that

$$
\mu\left(q \partial_{b(Q)} Q\right)=\mu(q)+\mu(Q)-b(Q) \epsilon_{\mu}(Q)<\mu\left(\partial_{b(Q)-j} Q\right)+\mu\left(\partial_{j} q\right)
$$

for all $j \in\{1, \ldots, b(Q)\}$.

Hence $\mu\left(\partial_{b(Q)}(q Q)\right)=\mu\left(\sum_{j=0}^{b(Q)}\left(\partial_{b(Q)-j} q \partial_{j} Q\right)\right)=\mu\left(q \partial_{b(Q)} Q\right)=\mu(q Q)-b(Q) \epsilon_{\mu}(Q)$.

On the other hand,

$$
\begin{array}{rrr}
\mu\left(\partial_{b(Q)}(q Q)\right) & = & \mu\left(\partial_{b(Q)}\left(P_{1} P_{2}\right)-\partial_{b(Q)}(r)\right) \\
& \geq & \min \left\{\mu\left(\partial_{b(Q)}\left(P_{1} P_{2}\right)\right), \mu\left(\partial_{b(Q)}(r)\right)\right\} \\
& \geq & \min \left\{\mu\left(\sum_{j=0}^{b(Q)} \partial_{j} P_{1} \partial_{b(Q)-j} P_{2}\right), \mu\left(\partial_{b(Q)} r\right)\right\} \\
& >\min _{0 \leq j \leq b(Q)}\left\{\mu\left(P_{1}\right)-j \epsilon_{\mu}(Q)+\mu\left(P_{2}\right)-(b(Q)-j) \epsilon_{\mu}(Q), \mu(r)-b(Q) \epsilon_{\mu}(Q)\right\} \\
& \geq & \mu(q Q)-b(Q) \epsilon_{\mu}(Q),
\end{array}
$$

which gives the desired contradiction. We have proved that $\mu\left(P_{1} P_{2}\right)=\mu(r)<\mu(q Q)$, so the Proposition holds in the case $t=2$.

Assume, inductively, that $t>2$ and that the Proposition is true for $t-1$. Let $P:=\prod_{i=1}^{t-1} P_{i}$. Let $P=q_{1} Q+r_{1}$ and $r_{1} P_{t}=q_{2} Q+r$ be the Euclidean divisions by $Q$ of $P$ and $r_{1} P_{t}$, respectivly. 
Note that $q=q_{1} P_{t}+q_{2}$. By the induction assumption, we have $\mu\left(r_{1}\right)=\mu(P)<\mu\left(q_{1} Q\right)$, hence $\mu\left(r_{1} P_{t}\right)=\mu\left(\prod_{i=1}^{t} P_{i}\right)<\mu\left(q_{1} P_{t} Q\right)$. By the case $t=2$ we have $\mu\left(r_{1} P_{t}\right)=\mu(r)<\mu\left(q_{2} Q\right)$. Hence $\mu(r)=\mu\left(r_{1} P_{t}\right)=\mu\left(\prod_{i=1}^{t} P_{i}\right)<\min \left\{\mu\left(q_{1} P_{t} Q\right), \mu\left(q_{2} Q\right)\right\} \leq \mu\left(q_{1} P_{t} Q+q_{2} Q\right)=\mu(q Q)$.

Definition 11. Let $Q$ be an abstract key polynomial for $\mu, g$ an element of $K[x]$ and $g=\sum_{j=0}^{s} g_{j} Q^{j}$ the $Q$-expansion of $g$. Put $S_{Q}(g)=\left\{j \in\{0, \ldots, s\}\right.$ such that $\left.\mu\left(g_{j} Q^{j}\right)=\mu_{Q}(g)\right\}$ and $\delta_{Q}(g):=\max S_{Q}(g)$.

Proposition 12. If $Q$ is an abstract key polynomial, then $\mu_{Q}$ is a valuation.

Proof. First, for any polynomials $f$ and $g$, we have

$$
\mu_{Q}(f+g) \geq \min \left\{\mu_{Q}(f), \mu_{Q}(g)\right\} .
$$

We want to show that

$$
\mu_{Q}(f g)=\mu_{Q}(f)+\mu_{Q}(g) .
$$

If both $f$ and $g$ have degree strictly less than $\operatorname{deg}(Q)$, we have $\mu_{Q}(f)=\mu(f)$ and $\mu_{Q}(g)=\mu(g)$. Furthermore, by Proposition 10, $\mu_{Q}(f g)=\mu(f g)$. Since $\mu$ is a valuation, (2.3) holds.

Next, let $i$, and $j$ be two non-negative integers. Let $f_{i}$ and $g_{j}$ be two polynomials of degree strictly less than $\operatorname{deg}(Q)$ and let $f_{i} g_{j}=a Q+b$ be the Euclidean division of $f_{i} g_{j}$ be $Q$. We have $\operatorname{deg} a, \operatorname{deg} b<\operatorname{deg} Q$ and

$$
\mu\left(f_{i} g_{j}\right)=\mu(b)<\mu(a Q)
$$

(by Proposition 10). Then $\left(f_{i} Q^{i}\right)\left(g_{j} Q^{j}\right)=a Q^{i+j+1}+b Q^{i+j}$ is a $Q$-expansion of $\left(f_{i} Q^{i}\right)\left(g_{j} Q^{j}\right)$. By definition of $\mu_{Q}$ and (2.4) we have

$$
\mu_{Q}\left(f_{i} g_{j} Q^{i+j}\right)=\mu\left(b Q^{i+j}\right)=\mu\left(f_{i} Q^{i}\right)+\mu\left(g_{j} Q^{j}\right)=\mu_{Q}\left(f_{i} Q^{i}\right)+\mu_{Q}\left(g_{j} Q^{j}\right),
$$

which proves the equality (2.3) for $f=f_{i} Q^{i}$ and $g=g_{j} Q^{j}$ with $\operatorname{deg} f_{i}$, $\operatorname{deg} g_{j}<\operatorname{deg} Q$.

It remains to show the equality (2.3) for arbitrary polynomials $f=\sum_{j=0}^{n} f_{j} Q^{j}$ and $g=$ $\sum_{j=0}^{m} g_{j} Q^{j}$. It is sufficient to consider the case when all the terms in the $Q$-expansion of $f$ have the same value and similarly for $g$. In other words, we may replace $f$ and $g$ by $\operatorname{In}_{\mathrm{Q}} f$ and $\operatorname{In}_{\mathrm{Q}} g$, respectively. By (2.2), (2.5) and the distributive law, we have

$$
\mu_{Q}(f g) \geq \mu_{Q}(f)+\mu_{Q}(g) .
$$

It remains to show that (2.6) is, in fact, an equality. Let $n_{0}:=\min S_{Q}(f)$ and $m_{0}:=$ $\min S_{Q}(g)$. We denote by

$$
f_{n_{0}} g_{m_{0}}=q Q+r
$$

the $Q$-expansion of $f_{n_{0}} g_{m_{0}}$. Hence the $Q$-expansion of $\operatorname{In}_{Q}(f) \operatorname{In}_{Q}(g)$ contains the term $r Q^{n_{0}+m_{0}}$, which, by Proposition 10, is of value

$$
\mu_{Q}\left(r Q^{n_{0}+m_{0}}\right)=\mu\left(r Q^{n_{0}+m_{0}}\right)=\mu\left(f_{n_{0}} Q^{n_{0}} g_{m_{0}} Q^{m_{0}}\right)=\mu_{Q}(f)+\mu_{Q}(g)
$$

. This completes the proof. 
Remark 13. Let $\alpha:=\operatorname{deg}_{x} Q$. We define $G_{<\alpha}:=\sum_{\operatorname{deg}_{x} P<\alpha}\left(\operatorname{in}_{\mu_{Q}} P\right) \operatorname{gr}_{\mu} K \subset \operatorname{gr}_{\mu_{Q}} K[x]$. It follows from the $t=2$ case of Proposition 10 that $G_{<\alpha}$ is closed under multiplication, so it is, in fact, a ring. The $\operatorname{ring} G_{<\alpha}$ embeds into $\operatorname{gr}_{\mu} K[x]$ by the natural map which sends $\operatorname{in}_{\mu_{Q}} f$ to $\operatorname{in}_{\mu} f$ for each polynomial $f$ of degree strictly less than $\alpha$. We have

$$
\operatorname{gr}_{\mu_{Q}} K[x]=G_{<\alpha}\left[\operatorname{in}_{\mu_{Q}} Q\right],
$$

where $\operatorname{in}_{\mu_{Q}} Q$ is transcendental over $G_{<\alpha}$. In particular, $\operatorname{in}_{\mu_{Q}} Q$ is irreducible in $\operatorname{gr}_{\mu_{Q}} K[x]$.

Lemma 14. For every polynomial $f \in K[x]$ and every $b \in \mathbb{N}^{*}$ we have

$$
\mu_{Q}\left(\partial_{b} f\right) \geq \mu_{Q}(f)-b \epsilon_{\mu}(Q)
$$

Proof. Let $f=\sum_{j=0}^{s} f_{j} Q^{j}$ be the $Q$-expansion of $f$.

It is enough to show the result for $f=f_{j} Q^{j}$. Indeed, if we have the result in this case, then

$$
\begin{aligned}
\mu_{Q}\left(\partial_{b} f\right) & =\mu_{Q}\left(\sum_{j=0}^{s} \partial_{b}\left(f_{j} Q^{j}\right)\right) \\
& \geq \min _{0 \leq j \leq s}\left\{\mu_{Q}\left(\partial_{b}\left(f_{j} Q^{j}\right)\right)\right\} \\
& \geq \min _{0 \leq j \leq s}\left\{\mu_{Q}\left(f_{j} Q^{j}\right)-b \epsilon_{\mu}(Q)\right\} \\
& \geq \min _{0 \leq j \leq s}\left\{\mu_{Q}\left(f_{j} Q^{j}\right)\right\}-b \epsilon(Q) \\
& \geq \mu(f)-b \epsilon_{\mu}(Q) .
\end{aligned}
$$

Now, let us show the result for $f=f_{j} Q^{j}$. First, we show it for $f=f_{j}$.

Indeed, $\epsilon_{\mu_{Q}}\left(f_{j}\right)=\epsilon_{\mu}\left(f_{j}\right)<\epsilon_{\mu}(Q)$ since $Q$ is an abstract key polynomial of degree strictly superior than $\operatorname{deg}\left(f_{j}\right)$. Hence

$$
\mu_{Q}\left(\partial_{b} f_{j}\right)=\mu\left(\partial_{b} f_{j}\right)>\mu_{Q}\left(f_{j}\right)-b \epsilon_{\mu}(Q) .
$$

This proves the Lemma with $f$ replaced by $f_{j}$.

We have $\mu_{Q}\left(\partial_{b} Q\right) \geq \mu_{Q}(Q)-b \epsilon_{\mu}(Q)$.

To finish the proof of the Lemma, it remains to show that if we have the result for two polynomials $f$ and $g$, we have the result for the product $f g$. Let us suppose that we have the result for two polynomials $f$ and $g$.

Then,

$$
\begin{array}{rcc}
\mu_{Q}\left(\partial_{b}(f g)\right) & = & \mu_{Q}\left(\sum_{s=0}^{b} \partial_{s} f \partial_{b-s} g\right) \\
\geq & \min _{0 \leq s \leq b}\left\{\mu_{Q}\left(\partial_{s} f\right)+\mu_{Q}\left(\partial_{b-s}(g)\right)\right\} \\
\geq & \min _{0 \leq s \leq b}\left\{\mu_{Q}\left(\partial_{s} f\right)\right\}+\min _{0 \leq s \leq b}\left\{\mu_{Q}\left(\partial_{b-s}(g)\right)\right\} \\
\geq & \mu_{Q}(f)-s \epsilon_{\mu}(Q)+\mu_{Q}(g)-(b-s) \epsilon_{\mu}(Q) \\
\geq & \mu_{Q}(f g)-b \epsilon_{\mu}(Q)
\end{array}
$$

This completes the proof.

Proposition 15. Let the notation be as in Definition 11. If $S_{Q}(g) \neq\{0\}$ then there exists $b \in \mathbb{N}^{*}$ such that $\frac{\mu_{Q}(g)-\mu_{Q}\left(\partial_{b} g\right)}{b}=\epsilon_{\mu}(Q)$.

Proof. First, replacing $g$ by $\operatorname{In}_{Q}(g)=\sum_{j \in S_{Q}(g)} g_{j} Q^{j}$ does not change the problem. We want to show the existence of a strictly positive integer $b$ such that $\mu_{Q}\left(\partial_{b} g\right)=\mu_{Q}(g)-b \epsilon_{\mu}(Q)$. 
Let $l$ be the minimum of $S_{Q}(g) \backslash\{0\}$. Write $l=p^{e} u$, with $p \nmid u$. Let $b:=p^{e} b(Q) \in \mathbb{N}^{*}$. We calculate $\partial_{b} g$.

Lemma 16. We have $\partial_{b} g=\operatorname{ur} Q^{l-p^{e}}+Q^{l-p^{e}+1} R+S$, where:

(1) $r$ is the remainder of the Euclidean division of $g_{l}\left(\partial_{b(Q)} Q\right)^{p^{e}}$ by $Q$.

(2) $R \in K[x]$.

(3) $S \in K[x]$ and $\mu_{Q}(S)>\mu_{Q}(g)-b \epsilon_{\mu}(Q)$.

Proof. First, let us show that the Lemma holds for $g=g_{l} Q^{l}$ and that for every integer $j \in S_{Q}(g) \backslash\{l\}$, we have $\partial_{b}\left(g_{j} Q^{j}\right)=Q^{l-p^{e}+1} R_{j}+S_{j}$, where $\left(R_{j}, S_{j}\right) \in K[x]^{2}$ such that $\mu_{Q}\left(S_{j}\right)>\mu_{Q}(g)-b \epsilon_{\mu}(Q)$.

Let us prove these two statements together.

First, put $M_{j}:=\left\{\left(b_{0}, \ldots, b_{s}\right) \in \mathbb{N}^{s+1}, b_{0}+\ldots+b_{s}=b, s \leq j\right\}$.

By the Leibnitz rule,

$$
\partial_{b}\left(g_{j} Q^{j}\right)=\sum_{\left(b_{0}, \ldots, b_{s}\right) \in M_{j}}(\underbrace{C\left(b_{0}, \ldots, b_{s}\right) \partial_{b_{0}} g_{j}\left(\prod_{i=1}^{s} \partial_{b_{i}} Q\right) Q^{j-s}}_{:=T\left(b_{0}, \ldots, b_{s}\right)}) . \text { where } C\left(b_{0}, \ldots, b_{s}\right)
$$

are certain integers whose exact values can be found in [2]. Here by "integer" we mean an element of the image of the natural map $\mathbb{N} \rightarrow K$, that is, an element of $\mathbb{N}$ or $\mathbb{F}_{p}$ depending on whether the characteristic of $K$ is 0 or $p>0$.

Put $N_{j}:=\left\{\left(b_{0}, \ldots, b_{s}\right) \in M_{j}\right.$ such that $b_{0}>0$ or $\left.\left\{b_{1}, \ldots, b_{s}\right\} \nsubseteq I(Q)\right\}$,

$S_{j}:=\sum_{\left(b_{0}, \ldots, b_{s}\right) \in N_{j}} T\left(b_{0}, \ldots, b_{s}\right), \alpha:=(0, \underbrace{b(Q), \ldots, b(Q)}_{p^{e}})$ and

$$
Q^{l-p^{e}+1} R_{j}:=\left\{\begin{array}{cl}
\sum_{\left(b_{0}, \ldots, b_{s}\right) \in M_{j} \backslash N_{j}} T\left(b_{0}, \ldots, b_{s}\right) & \text { if } j \neq l \\
\sum_{\left(b_{0}, \ldots, b_{s}\right) \in M_{j} \backslash N_{j}} T\left(b_{0}, \ldots, b_{s}\right) & \text { if } j=l \\
\left(b_{0}, \ldots, b_{s}\right) \neq \alpha &
\end{array}\right.
$$

If $j=l$, the number of times the term $T(0, \underbrace{b(Q), \ldots, b(Q)}_{p^{e}})$ appears in $\partial_{b}\left(g_{l} Q^{l}\right)$ is $\left(\begin{array}{c}l \\ p^{e}\end{array}\right)=u$.

Performing the Euclidean division of $T(0, \underbrace{b(Q), \ldots, b(Q)}_{p^{e}})$ by $Q$, we obtain

$$
T(0, \underbrace{b(Q), \ldots, b(Q)}_{p^{e}})=R_{0} Q^{l-p^{e}+1}+u r Q^{l-p^{e}}
$$

We are now in the position to calculate $\partial_{b} g$ : 


$$
\begin{aligned}
\partial_{b} g & = \\
& =\quad \partial_{b}\left(\sum_{b}\left(g_{l} Q^{l}\right)+\sum_{j \in S_{Q}(g)} g_{j} Q^{j}\right) \\
& =u r Q^{l-p^{e}}+Q^{l-p^{e}+1} R_{l}+S_{l}+\sum_{j \in S_{Q}(g) \backslash\{l\}}\left(g_{j} Q^{j}\right) \\
& =u r Q^{l-p^{e}}+Q^{l-p^{e}+1}(\underbrace{R_{l}+\sum_{j \in S_{Q}(g) \backslash\{l\}} R_{j}}_{:=R})+\underbrace{S_{l}+\sum_{j \in S_{Q}(g) \backslash\{l\}} S_{j}}_{:=S}
\end{aligned}
$$

with

$$
\begin{array}{rlrl}
\mu_{Q}(S) & \geq \min \left\{\mu_{Q}\left(S_{l}\right), \mu_{Q}\left(\sum_{j \in S_{Q}(g) \backslash\{l\}} S_{j}\right)\right\} \\
& \geq \\
& >\min _{j \in S_{Q}(g)}\left\{\mu_{Q}\left(S_{j}\right),\right\} \\
\mu_{Q}(g)-b \epsilon_{\mu}(Q) .
\end{array}
$$

This completes the proof of the Lemma.

Next, in view of Lemma 14, we have $\mu_{Q}\left(\partial_{b} g\right) \geq \mu_{Q}(g)-b \epsilon_{\mu}(Q)$.

Hence the $Q$-expansion of $\partial_{b} g$ contains the term $u r Q^{l-p^{e}}$ and terms wich either are divisible by $Q^{l-p^{e}+1}$ or have value greater than $\mu_{Q}(g)-b \epsilon_{\mu}(Q)$. To complete the proof of the Proposition, it is sufficient to show that $\mu_{Q}\left(u r Q^{l-p^{e}}\right)=\mu_{Q}\left(r Q^{l-p^{e}}\right)=\mu_{Q}(g)-b \epsilon_{\mu}(Q)$.

By Proposition 10 we have $\mu(r)=\mu_{Q}(r)=\mu\left(g_{l}\left(\partial_{b(Q)} Q\right)^{p^{e}}\right)$, hence

$$
\begin{aligned}
\mu_{Q}\left(r Q^{l-p^{e}}\right) & = & \mu\left(r Q^{l-p^{e}}\right) & =\mu\left(g_{l}\left(\partial_{b(Q)} Q\right)^{p^{e}} Q^{l-p^{e}}\right) \\
& =\mu\left(g_{l} Q^{l}\right)+p^{e} \mu\left(\partial_{b(Q)} Q\right)-p^{e} \mu(Q) & = & \mu\left(g_{l} Q^{l}\right)-p^{e} b(Q) \epsilon_{\mu}(Q) \\
& = & \mu_{Q}(g)-b \epsilon_{\mu}(Q) . &
\end{aligned}
$$

This completes the proof.

Remark 17. It can be shown that the implication of Proposition[15]is, in fact, an equivalence. This will be accomplished in a forthcoming paper.

Corollary 18. Let $Q$ be an abstract key polynomial and $f \in K[x]$. Suppose that there exists an integer $b \in \mathbb{N}^{*}$ such that $\frac{\mu_{Q}(f)-\mu_{Q}\left(\partial_{b} f\right)}{b}=\epsilon_{\mu}(Q)$ and $\mu_{Q}\left(\partial_{b} f\right)=\mu\left(\partial_{b} f\right)$. Then $\epsilon_{\mu}(f) \geq \epsilon_{\mu}(Q)$.

If moreover we have $\mu(f)>\mu_{Q}(f)$, then $\epsilon_{\mu}(f)>\epsilon_{\mu}(Q)$.

Proof. We have $\epsilon_{\mu}(f) \geq \frac{\mu(f)-\mu\left(\partial_{b} f\right)}{b}=\frac{\mu(f)-\mu_{Q}\left(\partial_{b} f\right)}{b}=\frac{\mu(f)+b \epsilon_{\mu}(Q)-\mu_{Q}(f)}{b}$. This means that $\epsilon_{\mu}(f)=\epsilon_{\mu}(Q)+\frac{\mu(f)-\mu_{Q}(f)}{b} \geq \epsilon_{\mu}(Q)$. And if $\mu(f)>\mu_{Q}(f)$, then $\epsilon_{\mu}(f)>\epsilon_{\mu}(Q)$.

Proposition 19. The polynomial $Q$ is $\mu_{Q}$-irreducible.

Proof. Put $L:=\operatorname{Frac}\left(G_{<\alpha}\right)$ where $\alpha=\operatorname{deg}(Q)$. Assume that

$$
\left(\operatorname{in}_{\mu_{Q}} Q\right)\left(\operatorname{in}_{\mu_{Q}} c\right)=\left(\operatorname{in}_{\mu_{Q}} g\right)\left(\operatorname{in}_{\mu_{Q}} h\right) \in L\left[\operatorname{in}_{\mu_{Q}} Q\right] .
$$

Then there exists $\lambda \in L^{*}$, such that $\operatorname{in}_{\mu_{Q}} Q=\lambda \operatorname{in}_{\mu_{Q}} g$ or $\operatorname{in}_{\mu_{Q}} Q=\lambda \operatorname{in}_{\mu_{Q}} h$. Since all of $\operatorname{in}_{\mu_{Q}} Q$, $\operatorname{in}_{\mu_{Q}} c, \operatorname{in}_{\mu_{Q}} g, \operatorname{in}_{\mu_{Q}} h$ are homogeneuos elements of $G_{<\alpha}\left[\operatorname{in}_{\mu_{Q}} Q\right]$, so is $\lambda$. This proves that $Q$ is $\mu_{Q^{-}}$irreducible. 
Proposition 20. Let $Q$ and $Q^{\prime}$ be abstract key polynomials such that $\epsilon_{\mu}(Q) \leq \epsilon_{\mu}\left(Q^{\prime}\right)$ and let $f \in K[x]$.

Then $\mu_{Q}(f) \leq \mu_{Q^{\prime}}(f)$. If $\mu_{Q}(f)=\mu(f)$, then $\mu_{Q^{\prime}}(f)=\mu(f)$.

Proof. First, we show that $\mu_{Q^{\prime}}(Q)=\mu(Q)$. If $\operatorname{deg}_{x}(Q)<\operatorname{deg}_{x}\left(Q^{\prime}\right)$, this is clear. Otherwise, we have $\operatorname{deg}_{x}(Q)=\operatorname{deg}_{x}\left(Q^{\prime}\right)$, since $Q$ is an abstract key polynomial and $\epsilon_{\mu}(Q) \leq \epsilon_{\mu}\left(Q^{\prime}\right)$. Let us suppose that $\mu_{Q^{\prime}}(Q)<\mu(Q)$. Then $S_{Q^{\prime}}(Q) \neq\{0\}$. In view of Proposition [15] and Corollary [18, we have $\epsilon_{\mu}(Q)>\epsilon_{\mu}\left(Q^{\prime}\right)$, which is a contradiction.

Now let $f=\sum_{j=0}^{s} f_{j} Q^{j}$ be the $Q$-expansion of $f$. For each integer $j \in\{0, \ldots, s\}$, we have

$$
\mu_{Q^{\prime}}\left(f_{j} Q^{j}\right)=\mu_{Q^{\prime}}\left(f_{j}\right)+j \mu_{Q^{\prime}}(Q)=\mu_{Q^{\prime}}\left(f_{j}\right)+j \mu(Q) .
$$

Then, since $\operatorname{deg}_{x}\left(f_{j}\right)<\operatorname{deg}_{x}(Q) \leq \operatorname{deg}_{x}\left(Q^{\prime}\right)$, we have $\mu_{Q^{\prime}}\left(f_{j} Q^{j}\right)=\mu\left(f_{j}\right)+j \mu(Q)=\mu\left(f_{j} Q^{j}\right)$. Hence $\mu_{Q^{\prime}}(f) \geq \min _{0 \leq j \leq s}\left\{\mu_{Q^{\prime}}\left(f_{j} Q^{j}\right)\right\}=\min _{0 \leq j \leq s}\left\{\mu\left(f_{j} Q^{j}\right)\right\}=\mu_{Q}(f)$.

Let us now suppose that $\mu_{Q}(f)=\mu(f) \leq \mu_{Q^{\prime}}(f)$. As we know that $\mu_{Q^{\prime}}(f) \leq \mu(f)$, we obtain

as desired.

$$
\mu_{Q}(f)=\mu_{Q^{\prime}}(f)
$$

Proposition 21. Let $f_{1}, \ldots, f_{r} \in K[x]$ be polynomials and let $n:=\max _{1 \leq i \leq r}\left\{\operatorname{deg}_{x}\left(f_{i}\right)\right\}$.

Then there exists an abstract key polynomial $Q$ of degree less than or equal to $n$ such that for each integer $i \in\{1, \ldots, r\}$, we have $\mu_{Q}\left(f_{i}\right)=\mu\left(f_{i}\right)$.

Proof. First, we show that it is sufficient to prove the Proposition for $r=1$.

Indeed, suppose the Proposition proved when there is just one polynomial and suppose $r>1$. Hence we can find $Q_{1}, \ldots, Q_{r}$ abstract key polynomials of degrees less or equal than $n$ such that for each integer $i \in\{1, \ldots, r\}$, we have $\mu_{Q_{i}}\left(f_{i}\right)=\mu\left(f_{i}\right)$.

Renumbering the $Q_{i}$, if necesssary, we may assume that $\epsilon_{\mu}\left(Q_{r}\right) \geq \epsilon_{\mu}\left(Q_{i}\right)$ for every integer $i \in\{1, \ldots, r\}$. By Proposition 20, we have, for each $i \in\{1, \ldots, r\}, \mu_{Q_{r}}\left(f_{i}\right)=\mu\left(f_{i}\right)$.

Let us show the case $r=1$. We argue by contradiction. Suppose that there exists a polynomial $f$ such that for every abstract key polynomial $Q$ of degree less than or equal to $\operatorname{deg}_{x}(f)$, we have $\mu_{Q}(f)<\mu(f)$. Choose $f$ of minimal degree among the polynomials having this property.

Claim. There exists an abstract key polynomial $Q$ of degree less than or equal to $\operatorname{deg}_{x} f$ such that

for every $b \in \mathbb{N}^{*}$.

$$
\mu_{Q}\left(\partial_{b} f\right)=\mu\left(\partial_{b} f\right)
$$

Indeed, let $s=\operatorname{deg}_{x} f$, so that for each integer $j$ strictly greater than $s$, we have $\partial_{j} f=0$. By the minimality assumption on $\operatorname{deg}_{x} f$, for each $i \in\{1, \ldots, s\}$ there exists an abstract key polynomial $Q_{i}$ such that $\mu_{Q_{i}}\left(\partial_{j} f\right)=\mu\left(\partial_{j} f\right)$,.

Take an $i \in\{1, \ldots, s\}$ such that $\epsilon_{\mu}\left(Q_{i}\right)=\max _{1 \leq j \leq s}\left\{\epsilon_{\mu}\left(Q_{j}\right)\right\}$. Then, in view of Proposition 20, for each integer $1 \leq j \leq s$, we have $\mu_{Q_{i}}\left(\partial_{j} f\right)=\mu\left(\partial_{j} f\right)$, and the Claim follows.

Now, we have $\mu_{Q}(f)<\mu(f)$, so in particular $S_{Q}(f) \neq\{0\}$, and for each $b \in \mathbb{N}^{*}, \mu_{Q}\left(\partial_{b} f\right)=$ $\mu\left(\partial_{b} f\right)$. In view of Proposition [15] and Corollary [18, we have

$$
\epsilon_{\mu}(f)>\epsilon_{\mu}(Q) \text {. }
$$


We claim that the last inequality is true for every abstract key polynomial of degree less than or equal to $\operatorname{deg}_{x} f$.

Indeed, let us take $Q^{\prime}$ an abstract key polynomial of degree less than or equal to $\operatorname{deg}_{x} f$. We have two cases.

First case: $\epsilon_{\mu}\left(Q^{\prime}\right) \leq \epsilon_{\mu}(Q)$. In view of (2.7), we have $\epsilon_{\mu}\left(Q^{\prime}\right) \leq \epsilon_{\mu}(Q)<\epsilon_{\mu}(f)$.

Second case: $\epsilon_{\mu}(Q)<\epsilon_{\mu}\left(Q^{\prime}\right)$. In view of Proposition 20, we have $\mu\left(\partial_{b} f\right)=\mu_{Q}\left(\partial_{b} f\right)=$ $\mu_{Q^{\prime}}\left(\partial_{b} f\right)$ for each strictly positive integer $b$. Since $\mu_{Q^{\prime}}(f)<\mu(f)$, arguing as before, we have $\epsilon_{\mu}\left(Q^{\prime}\right)<\epsilon_{\mu}(f)$.

By definition of the abstract key polynomials, there exists an abstract key polynomial $Q^{\prime}$ of degree less than or equal to $\operatorname{deg}_{x} f$ such that $\epsilon_{\mu}(f) \leq \epsilon_{\mu}\left(Q^{\prime}\right)$. This is a contradiction.

\section{The Relationship between the abstract and the Mac Lane-Vaquié key} POLYNOMIALS.

The aim of this section is to study the relationship between the abstract and the Mac Lane-Vaquié key polynomials.

Definition 22. Let $Q$ and $Q^{\prime}$ be two abstract key polynomials such that $\epsilon_{\mu}(Q)<\epsilon_{\mu}\left(Q^{\prime}\right)$. We say that $Q^{\prime}$ is an immediate successor of $Q$ and we write $Q<Q^{\prime}$ if $\operatorname{deg}_{x}\left(Q^{\prime}\right)$ is minimal among all the $Q^{\prime}$ which satisfy $\epsilon_{\mu}(Q)<\epsilon_{\mu}\left(Q^{\prime}\right)$.

Theorem 23. Let $Q$ be an abstract key polynomial for $\mu$. Then $Q$ is a Mac Lane - Vaquié key polynomial for $\mu_{Q}$.

Proof. We have to prove two things:

1. $Q$ is $\mu_{Q}$-irreducible.

2. $Q$ is $\mu_{Q}$-minimal.

Statement 1 is nothing but Proposition [19,

Now we are going to show the statement 2. We assume that $\left.Q\right|_{\mu_{Q}} r$, We want to show that $\operatorname{deg}_{x} r \geq \operatorname{deg}_{x} Q$.

By assumption, there exists $c$ such that

$$
\left(\operatorname{in}_{\mu_{\mathrm{Q}}} \mathrm{Q}\right)\left(\operatorname{in}_{\mu_{\mathrm{Q}}} \mathrm{c}\right)=\operatorname{in}_{\mu_{\mathrm{Q}}} \mathrm{r} \in \operatorname{gr}_{\mu_{Q}} K[x] \subset G_{<\alpha}\left[\operatorname{in}_{\mu_{Q}} Q\right] \subset L\left[\operatorname{in}_{\mu_{Q}} Q\right]
$$

. Since $\operatorname{in}_{\mu_{Q}} Q$ is transcendental over $L$, we have

$$
\operatorname{deg}_{\operatorname{in}_{\mu_{Q} Q}}\left(\text { in }_{\mu_{Q}} r\right) \geq 1 \text {. }
$$

Let $r=\sum_{j=0}^{n} r_{j} Q^{j}$ be the $Q$-expansion of $r$. By the algebraic independence of $\operatorname{in}_{\mu_{Q}} Q$ over $L$ (and hence, a fortiori, over $G_{<\alpha}$ ), we have $\operatorname{in}_{\mu_{Q}} r=\sum_{j=0}^{n} \operatorname{in}_{\mu_{Q}} r_{j} \operatorname{in}_{\mu_{Q}} Q^{j}$. combined with (3.1), this shows that $n \geq 1$. We obtain

$$
\operatorname{deg}_{x} r=n \operatorname{deg}_{x} Q+\operatorname{deg}_{x} r_{n} \geq \operatorname{deg}_{x} Q+\operatorname{deg}_{x} r_{n} \geq \operatorname{deg}_{x} Q .
$$

This completes the proof.

Lemma 24. Let $Q$ and $Q^{\prime}$ be two abstract key polynomials for $\mu$ such that $\epsilon_{\mu}(Q)<\epsilon_{\mu}\left(Q^{\prime}\right)$. Then

$$
\mu_{Q}\left(Q^{\prime}\right)<\mu\left(Q^{\prime}\right)
$$


Proof. In view of Lemma 14, we have $\frac{\mu_{Q}\left(Q^{\prime}\right)-\mu_{Q}\left(\partial_{b} Q^{\prime}\right)}{b} \leq \epsilon_{\mu}(Q)$ for each strictly positive integer b. Assume that $\mu_{Q}\left(Q^{\prime}\right)=\mu\left(Q^{\prime}\right)$, aiming for contradiction. Then

$$
\frac{\mu\left(Q^{\prime}\right)-\mu_{Q}\left(\partial_{b} Q^{\prime}\right)}{b} \leq \epsilon_{\mu}(Q)
$$

hence $\frac{\mu\left(Q^{\prime}\right)-\mu\left(\partial_{b} Q^{\prime}\right)}{b} \leq \epsilon_{\mu}(Q)$. In other words, $\epsilon_{\mu}\left(Q^{\prime}\right) \leq \epsilon_{\mu}(Q)$, which gives the desired contradiction.

Proposition 25. Let $Q$ and $Q^{\prime}$ be two abstract key polynomials for $\mu$. The following conditions are equivalent:

(1) $Q<Q^{\prime}$

(2) $\mu_{Q}\left(Q^{\prime}\right)<\mu\left(Q^{\prime}\right)$ and $Q^{\prime}$ is of minimal degree with respect to this property.

Proof. (2) $\Longrightarrow(1)$. Let us assume that $\mu_{Q}\left(Q^{\prime}\right)<\mu\left(Q^{\prime}\right)$ and that $Q^{\prime}$ is of minimal degree minimal for this property. Then $S_{Q}\left(Q^{\prime}\right) \neq\{0\}$ and for each strictly positive integer $b$, we have $\mu_{Q}\left(\partial_{b} Q^{\prime}\right)=\mu\left(\partial_{b} Q^{\prime}\right)$. By Proposition 15, there exists $b \in \mathbb{N}^{*}$ such that $\frac{\mu_{Q}\left(Q^{\prime}\right)-\mu_{Q}\left(\partial_{b} g\right)}{b}=\epsilon_{\mu}(Q)$. Hence $\frac{\mu_{Q}\left(Q^{\prime}\right)-\mu\left(\partial_{b} Q^{\prime}\right)}{b}=\epsilon_{\mu}(Q)$, and $\epsilon_{\mu}(Q)<\frac{\mu\left(Q^{\prime}\right)-\mu\left(\partial_{b} Q^{\prime}\right)}{b} \leq \epsilon_{\mu}\left(Q^{\prime}\right)$. If there exists a key polynomial $Q^{\prime \prime}$ satisfying $\epsilon_{\mu}(Q)<\epsilon_{\mu}\left(Q^{\prime \prime}\right)$ of degree strictly smaller than $\operatorname{deg}_{x} Q^{\prime}$, by Lemma 24 we would have $\mu_{Q}\left(Q^{\prime \prime}\right)<\mu\left(Q^{\prime \prime}\right)$, which would contradict the minimality assumption on the degree of $Q^{\prime}$. This proves (1).

$(1) \Longrightarrow(2)$. Let us assume that $Q<Q^{\prime}$. By Lemma 24, this implies that $\mu_{Q}\left(Q^{\prime}\right)<\mu\left(Q^{\prime}\right)$. Moreover, if there existed an abstract key polynomial $Q^{\prime \prime}$ satisfying $\mu_{Q}\left(Q^{\prime \prime}\right)<\mu\left(Q^{\prime \prime}\right)$ of degree strictly smaller than $\operatorname{deg}_{x} Q^{\prime}$, take such a $Q^{\prime \prime}$ of minimal degree. By the implication $(2) \Longrightarrow(1)$ of the Proposition we would have $\epsilon_{\mu}(Q)<\epsilon_{\mu}\left(Q^{\prime \prime}\right)$, which would contradict the minimality assumption on the degree of $Q^{\prime}$. This proves (2).

Theorem 26. Let $Q$ and $Q^{\prime}$ be two abstract key polynomials for $\mu$ such that $Q<Q^{\prime}$. Then $Q^{\prime}$ is a Mac Lane - Vaquié key polynomial for $\mu_{Q}$.

Proof. We have to prove two things:

1. $Q^{\prime}$ is $\mu_{Q}$-irreducible.

2. $Q^{\prime}$ is $\mu_{Q^{-}}$-minimal.

First we show 1. Let $\alpha=\operatorname{deg}_{x} Q$. By Remarks 8 and 13 , it is sufficient to show that $\operatorname{in}_{\mu_{Q}}\left(Q^{\prime}\right)$ is irreducible in

$$
G_{<\alpha}\left[\operatorname{in}_{\mu_{Q}}(Q)\right]=\operatorname{gr}_{\mu_{Q}} K[x] .
$$

Let $\varphi: \operatorname{gr}_{\mu_{Q}} K[x] \rightarrow \operatorname{gr}_{\mu} K[x]$ be the natural map which sends $\operatorname{in}_{\mu_{Q}}(f)$ to $\operatorname{in}_{\mu}(f)$ for every polynomial $f$. The map $\varphi$ maps $G_{<\alpha}$ isomorphically onto its image in $\operatorname{gr}_{\mu} K[x]$. The map $\varphi$ is not injective if and only if there exists a polynomial $f$ such that $\mu_{Q}(f)<\mu(f)$. In view of Proposition 25, we have this property for $f=Q^{\prime}$; in particular, $\operatorname{in}_{\mu_{Q}}\left(Q^{\prime}\right) \in \operatorname{Ker}(\varphi)$. We claim that $\operatorname{Ker}(\varphi)$ is a principal prime ideal, generated by $\operatorname{in}_{\mu_{Q}}\left(Q^{\prime}\right)$. Indeed, take any polynomial $f$ such that $\operatorname{in}_{\mu_{Q}} f \in \operatorname{Ker}(\varphi)$ and let $\operatorname{in}_{\mu_{Q}} f=\operatorname{in}_{\mu_{Q}}(a) \operatorname{in}_{\mu_{Q}}\left(Q^{\prime}\right)+\operatorname{in}_{\mu_{Q}}(r)$ be the Euclidean division of $\operatorname{in}_{\mu_{Q}} f$ by $\operatorname{in}_{\mu_{Q}}\left(Q^{\prime}\right)$. Then, if $\operatorname{in}_{\mu_{Q}}(r) \neq 0$ we have $\operatorname{in}_{\mu_{Q}}(r) \in \operatorname{Ker}(\varphi)$ and so $\mu_{Q}(r)<\mu(r)$, which contradicts the minimality of the degree of $Q^{\prime}$. Thus $\operatorname{Ker}(\varphi)=\left(\operatorname{in}_{\mu_{Q}}\left(Q^{\prime}\right)\right) \operatorname{gr}_{\mu_{Q}} K[x]$. Since gr ${ }_{\mu} K[x]$ has no zero divisors, we know that $\operatorname{in}_{\mu_{Q}}\left(Q^{\prime}\right)$ is a prime ideal. Thus $\operatorname{in}_{\mu_{Q}}\left(Q^{\prime}\right)$ is irreducible in $\operatorname{gr}_{\mu_{Q}} K[x]$. This completes the proof of 1 .

Now we show 2. Assume that $\left.Q^{\prime}\right|_{\mu_{Q}} r$. We want to show that $\operatorname{deg}_{x}(r) \geq \operatorname{deg}_{x}\left(Q^{\prime}\right)$. First, we know that $\operatorname{in}_{\mu_{Q}}\left(Q^{\prime}\right)$ divides $\operatorname{in}_{\mu_{Q}}(r)$ in the unique factorisation domain $L\left[\operatorname{in}_{\mu_{Q}} Q\right] \supset$ $\mathrm{G}_{<\alpha}\left[\operatorname{in}_{\mu_{Q}} Q\right]=\operatorname{gr}_{\mu_{Q}} K[x]$. Hence $r \in \operatorname{Ker}(\varphi)$. In other words, $\mu_{Q}(r)<\mu(r)$. On the other 
hand, we know that $\mu_{Q}\left(Q^{\prime}\right)<\mu\left(Q^{\prime}\right)$ and that $Q^{\prime}$ is of minimal degree for this property in view of Proposition 25. By the minimality of $\operatorname{deg}_{x}\left(Q^{\prime}\right)$, we get the result.

Theorem 27. Fix a monic polynomial $Q \in K[x]$. Let $\mu^{\prime}$ be a valuation of $K(x)$ such that:

1. For each $f$ of degree strictly less than $\operatorname{deg}(Q)$, we have $\mu^{\prime}(f)=\mu(f)$;

2. $\mu^{\prime}(Q)>\mu(Q)$.

Then $Q$ is an abstract key polynomial for $\mu^{\prime}$.

Proof. Assume that $Q$ is not an abstract key polynomial for $\mu^{\prime}$. Then there exists a monic polynomial $g$ such that

$$
\epsilon_{\mu^{\prime}}(g) \geq \epsilon_{\mu^{\prime}}(Q)
$$

and

$$
\operatorname{deg}(g)<\operatorname{deg}(Q) .
$$

We can choose $g$ of minimal degree for this property, and hence $g$ is an abstract key polynomial.

Thus there exists an abstract key polynomial $g$ such that $\epsilon_{\mu^{\prime}}(g) \geq \epsilon_{\mu^{\prime}}(Q)$ and $\operatorname{deg}(g)<$ $\operatorname{deg}(Q)$.

Since every derivative of $Q$ has degree strictly smaller than $\operatorname{deg}(Q)$, we have

$$
\operatorname{deg}_{x} g \leq \operatorname{deg}_{x}\left(\partial_{1} Q\right)=\max _{b \in \mathbb{N}^{*}}\left\{\operatorname{deg}_{x}\left(\partial_{b} Q\right)\right\} .
$$

By Proposition 21, replacing $g$ by another abstract key polynomial with larger $\epsilon_{\mu}$, if necessary, we may assume, in addition, that

$$
\mu^{\prime}\left(\partial_{b} Q\right)=\mu_{g}^{\prime}\left(\partial_{b} Q\right)
$$

for all strictly positive integers $b$ (at this point, the abstract key polynomial $g$ still satisfies (3.2) and (3.3) but we may no longer have the condition that $g$ is of minimal degree for this property).

We claim that for each polynomial $h$, we have

$$
\mu(h) \geq \mu_{g}^{\prime}(h) .
$$

Indeed, let $h=\sum_{j=0}^{l} h_{j} g^{j}$ be the $g$-expansion of $h$. We have

$$
\mu_{g}^{\prime}(h)=\mu_{g}^{\prime}\left(\sum_{j=0}^{l} h_{j} g^{j}\right)=\min _{0 \leq j \leq l}\left\{\mu^{\prime}\left(h_{j}\right)+j \mu^{\prime}(g)\right\}=\min _{0 \leq j \leq l}\left\{\mu\left(h_{j}\right)+j \mu(g)\right\}
$$

by hypothesis 1 .

Hence $\mu_{g}^{\prime}(h)=\min _{0 \leq j \leq l} \mu\left(h_{j} g^{j}\right) \leq \mu(h)$. In particular, $\mu^{\prime}(Q)>\mu(Q) \geq \mu_{g}^{\prime}(Q)$.

Recall that if $Q=\sum_{j=0}^{s} Q_{j} g^{j}$ is the $g$-expansion of $Q$, we denote

$$
S_{g}(Q)=\left\{j \in\{0, \ldots, s\} \mid \mu^{\prime}\left(Q_{j} g^{j}\right)=\mu_{g}^{\prime}(Q)\right\} .
$$

Suppose $S_{g}(Q)=\{0\}$, then $\mu^{\prime}(Q)>\mu_{g}^{\prime}(Q)=\mu^{\prime}\left(Q_{0}\right)$. Hence

$$
\mu^{\prime}\left(Q_{0}\right)=\mu^{\prime}\left(\sum_{j \geq 1} Q_{j} g^{j}\right) \geq \min _{j \geq 1} \mu^{\prime}\left(Q_{j} g^{j}\right)>\mu^{\prime}\left(Q_{0}\right)
$$

which is a contradiction. We have proved that $S_{g}(Q) \neq\{0\}$. 
By Proposition 15, there exists a strictly positive integer $b$ such that $\frac{\mu_{g}^{\prime}(Q)-\mu_{g}^{\prime}\left(\partial_{b} Q\right)}{b}=\epsilon_{\mu^{\prime}}(g)$. By virtue of (3.4) we obtain $\epsilon_{\mu^{\prime}}(g)=\frac{\mu_{g}^{\prime}(Q)-\mu_{g}^{\prime}\left(\partial_{b} Q\right)}{b}=\frac{\mu_{g}^{\prime}(Q)-\mu^{\prime}\left(\partial_{b} Q\right)}{b}$. Since $\mu^{\prime}(Q)>\mu_{g}^{\prime}(Q)$, we have

which is a contradiciton.

$$
\epsilon_{\mu^{\prime}}(g)<\frac{\mu^{\prime}(Q)-\mu^{\prime}\left(\partial_{b} Q\right)}{b}=\epsilon_{\mu^{\prime}}(Q)
$$

Hence $Q$ is an abstract key polynomial for $\mu^{\prime}$.

Proposition 28. (9], Proposition 1.3) Let $Q$ be a Mac Lane - Vaquié key polynomial for the valuation $\mu$. Then there exists a valuation $\mu^{\prime}$ such that:

(1) For each $f$ of degree strictly less than $\operatorname{deg}(Q)$, we have $\mu^{\prime}(f)=\mu(f)$

(2) $\mu^{\prime}(Q)>\mu(Q)$.

Corollary 29. Let $Q$ be a Mac Lane - Vaquié key polynomial for the valuation $\mu$. Then it is an abstract key polynomial for any valuation $\mu^{\prime}$ satisfying the conclusion of Proposition 28.

\section{REFERENCES}

[1] F. J. Herrera Govantes, M. A. Olalla Acosta, M. Spivakovsky, Valuations in algebraic field extensions, Journal of Algebra, Volume 312, Issue 2 (2007), pages 1033-1074.

[2] F. J. Herrera Govantes, W. Mahboub, M. A. Olalla Acosta, M. Spivakovsky Key polynomials for simple extensions of valued fields., arXiv:1406.0657v3 [math.AG].

[3] S. Mac Lane, A construction for prime ideals as absolute values of an algebraic field, Duke Math. J., vol 2 (1936), pages 492-510.

[4] S. MacLane A construction for absolute values in polynomial rings, Transactions of the AMS, vol 40 (1936), pages 363-395.

[5] W. Mahboub Key Polynomials, Journal of Pure and Applied Algebra, 217(6) (2013), pages 989-1006.

[6] W. Mahboub Une construction explicite de polynômes-clés pour des valuations de rang fini, Thèse de Doctorat, Institut de Mathématiques de Toulouse, 2013.

[7] J.-C. San Saturnino, Defect of an extension, key polynomials and local uniformization, preprint, arXiv:1412.7697, 2014.

[8] Vaquié, Michel, Famille admise associée à une valuation de K[x]. [Admissible family associated with a valuation of $K[x]$ /, Singularités Franco-Japonaises, 391-428, Sémin. Congr., 10, Soc. Math. France, Paris, 2005.

[9] Vaquié, Michel, Extension d'une valuation. [Extension of a valuation], Trans. Amer. Math. Soc. 359 (2007), no. 7, 3439-3481. (electronic)

[10] Vaquié, Michel, Famille admissible de valuations et défaut d'une extension. [Admissible family of valuations and defect of an extension], J. Algebra 311 (2007), no. 2, 859-876.

[11] Vaquié, Michel, Extensions de valuation et polygone de Newton. /Valuation extensions and Newton polygon] Ann. Inst. Fourier (Grenoble) 58 (2008), no. 7, 2503-2541. 